Calculated intensities :

\pm [pqr] (hkl) (002) (110) (112) (222)

[100]

[010]

[001]

[110]

[110]

[101]

[10̄̄]

[011]

[011]
Observed intensities :

(002) (110) (112) (222)

$-\quad-\quad-$

$-\rightarrow-$

$-\rightarrow-$

- V.S. M. S.

M.S. M. W. -

M.S. M. S. S.

M.S. M. W. -

M.S. M. S. S.

Sodium single crystals are very soft and elastically anisotropic; they are body-centred cubic in structure. Lead single erystals are also soft and are anisotropic in the same sense $\left(c_{11}-c_{12}<2 c_{44}\right)$, although the shear constant $c_{44}$ is much smaller relative to the compressibility $\left(c_{11}+2 c_{12}\right)$; but they are face-centred cubic in structure. Diffuse reflexion photographs (which are particularly good for large angles of incidence, on account of the slow decrease of the atomic scattering factor) show that the reflecting regions in reciprocal space are similar to those found for sodium, although the intensity 'spikes' are less pronounced. At low temperatures the diffuse reflexions almost disappear, but the Laue spots become much more numerous and the background clearer.

Tungsten crystallizes, like sodium, with a body. centred cubic structure, but it is elastically isotropic $\left(c_{11}-c_{12}=2 c_{44}\right)$ and the elastic constants are large.

\begin{tabular}{|c|c|c|c|c|}
\hline$c_{11}$ & $c_{1}$ & $c_{44}$ & $c_{11}-c_{1 \ell}$ & $c_{11}+2 c_{18}\left(\times 10^{11}\right.$ dynes $\left./ \mathrm{cm}_{*}{ }^{2}\right)$ \\
\hline $0 \cdot 52$ & 0.40 & 0.41 & $0 \cdot 12$ & $1 \cdot 32 \quad(4)$ \\
\hline $4 \cdot 77$ & $4 \cdot 03$ & $1 \cdot 44$ & 0.74 & $12 \cdot 83$ \\
\hline $\left.\begin{array}{l}51 \cdot 3 \\
50 \cdot 1\end{array}\right\}$ & $\left.\begin{array}{l}20 \cdot 6 \\
19 \cdot 8\end{array}\right\}$ & $\left.\begin{array}{c}15 \cdot 3 \\
15 \cdot 1\end{array}\right]$ & $\left.\begin{array}{l}30 \cdot 7 \\
30 \cdot 3\end{array}\right\}$ & $\left.\begin{array}{l}92 \cdot 5 \\
89 \cdot 7\end{array}\right\}$ \\
\hline
\end{tabular}

For tungsten, the Jahn formula predicts small, elliptical diffuse spots which should disappear at a very small angular distance from the Bragg position. This is exactly what we find. The diffuse spots are smaller than the Laue spots, and disappear at $\theta_{B} \pm 2^{\circ}$; there are none of the detailed, persistent groups of spots and streaks (some of which extend right across the Brillouin zone boundaries) that are found for both sodium and lead. A rough calcula. tion, which is in good qualitative agreement with our observation, indicates that the tungsten diffuse reflexion should be about so, and that of lead about $\frac{1}{2}$, as intense as the diffuse reflexion from sodium, for the first observed order from (110) planes.

The thermal theory predicts that the relation of diffuse to normal (Bragg) reflecting power for any set of planes should be governed by the actual values of the elastic constants and not primarily by the crystal structure. In our opinion, this is proved by the above experiments on single crystals of metals.

K. LONSDALE.

Royal Institution,

H. Sмттн.

Albernarle Street, London, W.I.

1 Lonsdale and Smith, NATORE, 148, 628 (1941).

-Waller, "Theoretische Studien zur Interferenz- und Dispersions. theorie der Röntgenstrahlen" (Uppsala: Universitets Arsskrift, 1925).

Jahn, Nature, 147, 511 (1941); Proc. Roy. Soc., A (in the press).

Quimby and Siegel, Phys. Rev., 54, 299 (1938); data extrapolated to room temperature.

- Coens, Phys. 2., 37, 321 (1936); data corrected for an arithmetical error.

Bridgman, Proc. Amer. Acad., 60, 305 (1925).

"Wright, Proc. Roy. Soc., A, 126, 613 (1930).

\section{A Reversible Discharge Tube}

R. W. WOOD ${ }^{1}$ has described an interesting discharge tübe which could be made to exhibit "either the spectrum of atomic hydrogen or molecular oxygen at will" on excitation by a high-frequency discharge. In the course of investigations on the spectra of gases excited by high-frequency discharge, we have prepared a discharge tube which has an analogous behaviour and can be made to show either the air spectrum or hydrogen spectrum as desired. The fundamental difference between the two tubes, however, is that under the discharge hydrogen is suppressed by oxygen in Wood's tube, whereas here, hydrogen suppresses air.

The discharge tube, which has a quartz window and aluminium electrodes, was sealed off the evacua tion apparatus after being repeatedly washed by hydrogen. The spectrogram taken soon afterwards showed only the spectra of hydrogen, in particular a very intense development of the well-known continuous spectrum of hydrogen. The tube was excited by high-frequency discharge. On standing for a few days the tube, on similar excitation, showed only nitrogen bands in the visible, clearly due to traces of residual air, and there was no trace of hydrogen. On continued excitation, however, hydrogen gradually appeared, nitrogen got weaker and in a couple of hours the tube showed nothing but hydrogen, nitrogen being suppressed under the discharge. The continuous spectrum of hydrogen was found to be considerably weakened in intensity while the atomic spectrum was very prominent, due, no doubt, to the absorption of hydrogen by the walls of the tube resulting in a gradual 'clean up'.

On cutting off the discharge and allowing the tube to rest for some period, the same series of phenomena can be repeated. Most of the hydrogen appears to be confined to the electrodes, for it has been possible so to excite the tube with external electrodes that, by omitting the internal electrodes from the path of discharge, the tube shows only the spectrum of air for any length of time.

R. K. AsUndI.

NaND LAL SINGH.

JAG Deo Srngh.

Benares Hindu University, Oct. 25.

${ }^{2}$ Phys. Rev., 35, 658 (1930).

\section{Psycho-Physical Significance of the Dissipation Coefficient of Soft Materials}

IN an earlier communication ${ }^{1}$ we raised the question of the significance of the dissipation coefficient (k) of soft materials, which we measure by compressing eylinders under loads compensated to ensure constant shear stress $(S)$. For materials the firmness $(\psi)$ of which is independent of stress, $k$ is defined by an equation of the Nutting type ${ }^{2}$

$$
\psi=S \sigma^{-1} t k
$$

where $\sigma$ is shear strain and $t$ is time.

Broome and Bilmes have shown ${ }^{3}$ that $k$ is also given by the ratio of mean to differential viscosity $\left.(S) \frac{\sigma}{t} \div S / \frac{\partial \sigma}{\partial t}\right)$ and, for relaxation at constant strain, differentiation shows that the Maxwell relaxation time will vary as a power $(1 / k)$ of the stress. 